\title{
Individual Protective Covers (IPCs) for Young Tree Protection from the HLB Vector, the Asian Citrus Psyllid ${ }^{1}$
}

\author{
Fernando Alferez, Ute Albrecht, Susmita Gaire, Ozgur Batuman, Jawwad Qureshi, and Mongi \\ Zekri $^{2}$
}

\section{Rationale}

Because of the endemic presence of huanglongbing (HLB) and its vector, the Asian citrus psyllid (ACP), in Florida, there is a strong need for planting new citrus trees to replace those that have become unproductive. Citrus in Florida faces unique challenges as mature infected trees coexist with newly planted healthy trees that need to be protected from ACP and HLB but have different physiologies and require different management. In mature trees, the main goal is to sustain fruit production, because trees are already infected with Candidatus Liberibacter asiaticus (CLas), the bacterium associated with HLB. In contrast, young trees are HLB-free at planting, and the main goal is to keep them healthy and free from the disease as long as possible, or at least until they bear fruit. ACP infestation in young trees is usually managed by soil drenches of systemic insecticides rotated with foliar sprays of insecticides with a different mode of action (MOA).

Psyllid exclusion is the most effective strategy to keep citrus trees free from HLB. Citrus Under Protective Screens (CUPS) have been proven effective for growing trees in an enclosed environment and keeping them disease-free (Schumann et al. 2020). However, this system is expensive and probably most applicable for smaller acreages of specialty crops, such as fresh-fruit varieties with a high return on investment. Individual protective covers (IPCs) are a novel strategy based on psyllid exclusion of individual trees using a protective mesh bag. IPCs are economically more feasible for processing varieties grown on a large acreage in Florida. The IPC system provides an alternative to soil drenches and foliar insecticides, which effectively reduce psyllid populations but cannot always prevent $C$ Las infection. The situation is exacerbated by the increasing levels of psyllid resistance to the neonicotinoid insecticides, which have been used extensively for more than a decade to protect young trees from ACP. Psyllid exclusion by using IPCs is therefore a promising tool that has sparked interest in recent years, with many growers adopting this technology in their groves.

This document is of special interest for growers planting new trees at their groves. Here we summarize the knowledge we have accrued from our 3-year-long field studies using the IPC system and provide recommendations that can help with effective use of this technology.

\section{IPC Characteristics and Installation}

Currently, there is a wide variety of models and sizes for IPCs. In general, IPCs are net covers that are made

1. This document is HS1425, one of a series of the Horticultural Sciences Department, UF/IFAS Extension. Original publication date October 2021. Visit the EDIS website at https://edis.ifas.ufl.edu for the currently supported version of this publication.

2. Fernando Alferez, assistant professor; Ute Albrecht, assistant professor; Susmita Gaire, graduate student, Horticultural Sciences Department; Ozgur Batuman, assistant professor, Plant Pathology Department; Jawwad Qureshi, associate professor, Entomology and Nematology Department, UF/IFAS Southwest Florida REC; and Mongi Zekri, citrus Extension agent IV, UF/IFAS Extension Hendry County; UF/IFAS Extension, Gainesville, FL 32611.

The Institute of Food and Agricultural Sciences (IFAS) is an Equal Opportunity Institution authorized to provide research, educational information and other services

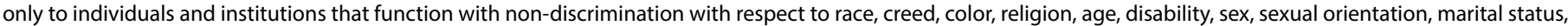


U.S. Department of Agriculture, UF/IFAS Extension Service, University of Florida, IFAS, Florida A \& M University Cooperative Extension Program, and Boards of County Commissioners Cooperating. Nick T. Place, dean for UF/IFAS Extension. 
of monofilament high-density polyethylene (HDPE) or polyvinyl with a mesh size of $50(0.297 \mathrm{~mm}$ holes, i.e., 50 holes per linear inch), which is small enough to exclude psyllids from the trees, because the average width of a psyllid adult is $0.57 \mathrm{~mm}$. IPC sizes vary from 3 feet to 8 feet or more, and depending on the size, a PVC pole with or without spreaders must also be installed to serve as support for the net. The net covers are tightly secured to the trunk and PVC pole with zip ties just below the graft union; the covers can be tucked into the tree wraps and secured there, or they can be secured outside the wraps (Figure 1).
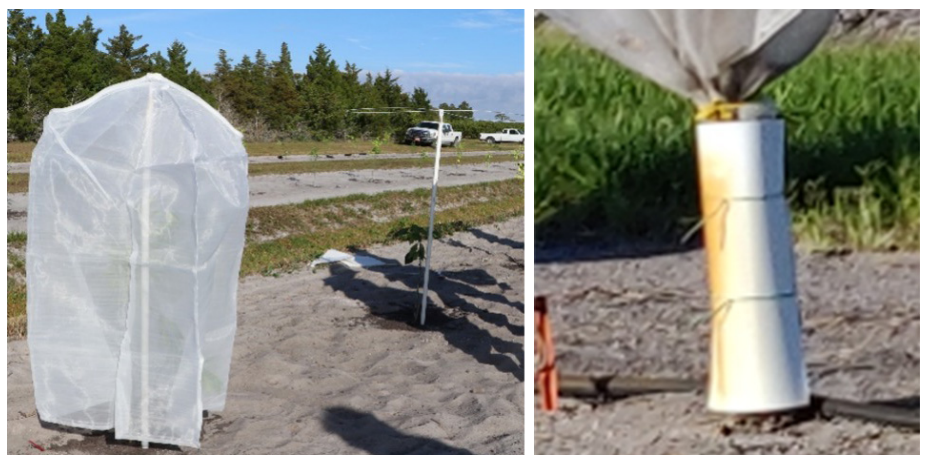

Figure 1. Installation of IPCs. Detail showing PVC pole and spreader (left) and trunk protection holding the IPC close to the trunk and tucked into the wrap (right).

Credits: Susmita Gaire, UF/IFAS

IPCs can be installed on solid blocks of trees or in resets (Figure 2). As mentioned above, IPCs are especially valuable for planting reset trees in gaps left by dead or removed trees in mature groves where HLB incidence is typically higher and the risk of infection is therefore greatest. IPCs should be placed immediately during planting to prevent any exposure of trees to the psyllids. Tree management can be performed as for noncovered trees, but psyllid management can be reduced or eliminated. Nevertheless, scouting for pest and diseases should be continued.

The period IPCs can stay on the trees varies with the rootstock/scion combination, grove management, age of the tree, and size of the cover. Multiple sizes of IPCs are now available to accommodate fast-growing trees on vigorous rootstocks or with vigorous scions; if the trees outgrow the smaller IPCs they can be replaced with larger ones to provide more time under protective cover. The right time to replace smaller IPCs with larger ones is when the canopy has completely filled the bag (typically around 2 years). Alternatively, the bags can be removed permanently at that time. The largest IPCs currently available are 8 feet in size.

\section{IPC Field Trials \\ Psyllid Exclusion}

In a 3-year field study, no HLB leaf symptoms were found on IPC-covered trees, and all trees tested negative for CLas. In contrast, all noncovered trees showed chlorosis and blotchy mottling of leaves, which are typical foliar symptoms of HLB, and consistently tested positive for CLas as early as 6 months after planting. This demonstrates that IPCs were effective in excluding psyllids and therefore preventing CLas transmission and disease development in young trees during the early years of tree establishment. Healthy vegetative growth after planting is essential for fruit production as the trees enter the productive stage and therefore substantially contribute toward the viability of a grove.

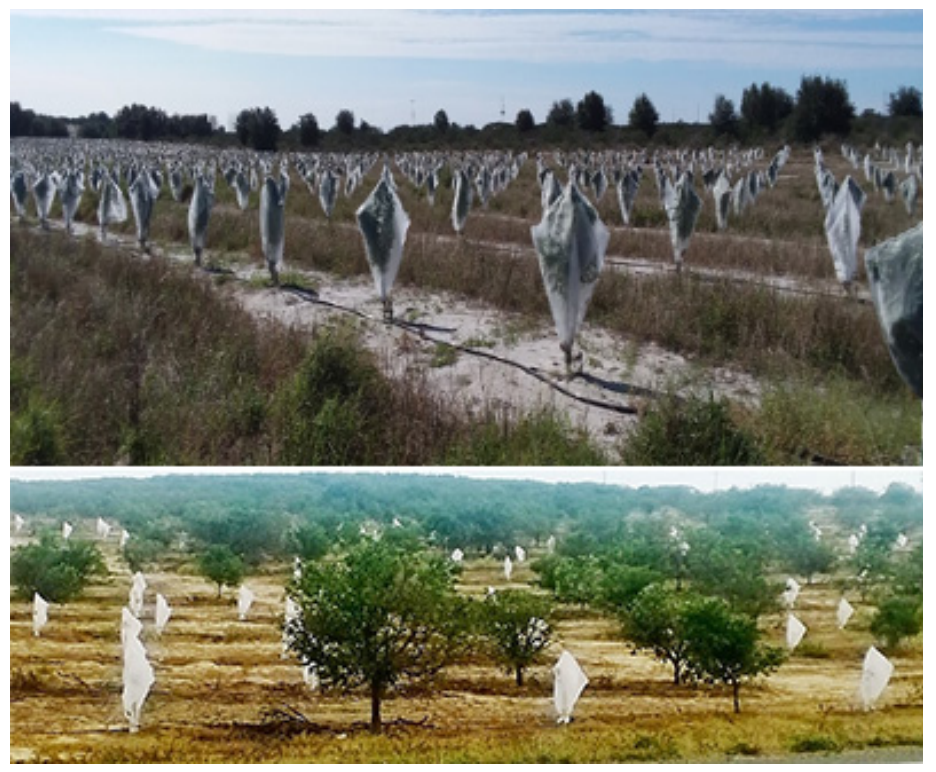

Figure 2. IPCs in a large-scale commercial "solid block" grove and on "resets."

Credits: F. Alferez (top) and M. Zekri (bottom), UF/IFAS

\section{Other Pests and Diseases}

In addition to excluding psyllids and preventing HLB, IPCs also reduced the incidence of citrus leaf miners. However, some other pests typically found on noncovered trees were also found within the covers. These pests included snow-, purple-, and black scales, mites, and armyworms. It appears that the armyworm moth was able to lay eggs on the foliage through the mesh, or on the mesh itself, allowing the first instars to move through the holes and resulting in some feeding damage. There have been some reports of mealybug infestation in IPC-covered trees in central Florida, but this was not confirmed in our studies in southwest Florida. This suggests that IPC management will have to be adjusted based on the geographic location and growing environment. We also found a lower incidence of 
canker in IPC-covered trees; however, greasy spot and sooty mold were somewhat more prevalent in trees with IPCs than in noncovered trees. Taken together, this means that IPCs do not exclude all pests, and that regular scouting and insecticide applications may still be necessary to manage other pests of citrus as growers would do on uncovered trees as part of general grove management. Scouting should be performed at least once every three months (seasonally) and particularly right after severe inclement weather events such as sustained forceful wind, hail, and hurricanes. Scouting is most effective if the trees are uncovered, but it is recommended to leave them uncovered for no more than a few minutes, typically a time needed for the grower to uncover, thoroughly inspect, and cover the tree again. Foliar sprays of insecticides done with a hoop sprayer or speed sprayer provide good coverage on the trees protected with IPCs.

\section{IPC Effects on Tree Growth}

IPCs modify the atmosphere within the covered canopy by altering relative humidity and temperature, resulting in a lower vapor pressure deficit (VPD) compared with noncovered trees. A lower VPD means that the air contains more moisture, which allows the leaf stomata to stay open longer and extend the length of time available for photosynthesis compared with higher-VPD conditions. Under IPCs, the leaves were greener and larger with more chlorophyll, more soluble sugars, and less starch, indicating that they have been metabolically more active than noncovered trees. Interestingly, trees under IPCs flushed earlier and more profusely, and flushing was more synchronized compared to trees without IPCs, which ultimately resulted in faster tree growth. Depending on the initial IPC size and the desired time of protection, smaller IPCs may therefore need to be replaced with larger ones to accommodate the growing canopy. After final IPC removal, tree care can commence using methods typical for the production site.

In summary, although IPCs are not a one-size-fits-all system for citrus pest and disease management, they are effective in excluding psyllids and preventing CLas infection during the most vulnerable early years of tree growth, provided they are properly installed and remain undamaged during their time of use. IPCs can serve as another tool for growers to manage HLB in young citrus trees but can promote other pests and diseases inside the covers if not managed. Thus, regular scouting and management will still be required to retain tree health and ensure productivity in the years to come. Because IPC is a novel citrus production system, other challenges (or positive effects) may emerge as it continues to be evaluated with different rootstock/ scion combinations, different installment patterns (e.g., protecting trees with IPC in "hot spots" such as in the grove perimeter, or in alternating patterns that are more cost friendly) and in different climatic conditions across Florida. The guidelines presented here are therefore preliminary and will be updated as soon as new information from our ongoing research trials becomes available.

\section{Suggested Further Reading (References)}

Schumann, Arnold W., Ariel Singerman, Alan L. Wright, Rhuanito S. Ferrarezi, Jawwad Qureshi, and Fernando Alferez. 2021. "2021-2022 Florida Citrus Production Guide: Citrus Under Protective Screen (CUPS) Production Systems." EDIS 2021 (CPG). https://doi.org/10.32473/ edis-hs1304-2021 